\title{
Gas Chromatographic Characterization of Silica-Based Reversed-Phase Sorbents for Solid Phase Extraction
}

\author{
Jung Hag Park ${ }^{\dagger}$ and Yeong Chun Weon \\ Department of Chemistry, College of Science, Yeungnam University, \\ Kyongsan 712-749, Korea
}

\begin{abstract}
The interaction properties of some alkylsiloxane-, phenylsiloxane- and cyanopropylsiloxane-bonded silica-based sorbents for solid phase extraction are characterized. The linear solvation energy relationship model is used to estimate the characteristic interaction constants of the sorbents, $s$ (dipolarity/polarizability), $a$ (hydrogen bonding basicity), $b$ (hydrogen bonding acidity), $r$ (polarizability), and $l$ (methylene selectivity). For nonpolar alkyl-bonded sorbents, the $l$ constant is the largest, indicating that the cavity/dispersive interactions term is the dominant term favoring retention on the sorbent. Other interaction terms play minor roles in retention. For the polar cyanopropyl-bonded sorbent the $s$ and $a$ constants are the largest and are essentially equal, followed by $l, r$ and $b$ constants. For the phenyl-bonded sorbent, the $b$ coefficient is the largest, followed by a quite large $a$ coefficient.
\end{abstract}

Keywords Characteristic interaction constant, reversed-phase sorbent, solid phase extraction, gas chromatography, linear solvation energy relationship

Solid phase extraction (SPE) is widely used to isolate and concentrate organic contaminants from water, largely replacing liquid-liquid extraction. ${ }^{1}$ A variety of silica-based sorbents with different bonded ligands are broadly used in SPE. Among these silica-based sorbents, reversed-phase sorbents with various bonded functional groups are widely used.

Practicing chromatographers have noted the significant differences in retention characteristics between nominally equivalent silica-based stationary phases for reversed phase liquid chromatography (RPLC) such as $\mathrm{C}-18^{2}$ and cyano-bonded materials. ${ }^{3}$ This variability comes naturally since retention in RPLC is determined by both solvophobic and chemical interactions between solute molecules and reactive sites of the stationary phase, which involve not only interactions between solute molecules and the organic bonded phase (solvophobic) ${ }^{4}$ but also hydrogen bonding interactions with unreacted silanol groups and complexation with trace metals on the silica surfaces. ${ }^{5}$ The relative contributions of these two types of interactions depends on the characteristics of the stationary phase, which include the nature of the base silica particles such as the specific surface area and pore size and volume, the nature of bonded organic ligand, and the bonding process. Chemical interactions are often regarded as undesirable since these types of interactions, in particular silanophilic interactions, are responsible for the excessive peak tailing and long retention times observed for basic solutes. The presence

$\dagger$ To whom correspondence should be addressed. of these types of interactions also leads to poorer control of the column packing, resulting in column-to-column irreproducibility.,5 As diversity in retention properties of various stationary phases due to this variability has caused many difficulties for chromatographers in selecting the best column for a given separation, knowledge of the nature and relative contributions of possible interaction between the solute and stationary phase to RPLC retention is of great value, in that it can be utilized to help chromatographers in the task of selecting the best column and optimizing the selectivity for a given separation. Recently $w^{6}{ }^{6}$ described a simple method of characterizing the stationary phase for RPLC in terms of the type and relative strength of the solutestationary phase interactions by multiple regression analysis of retention data for a set of test compounds based on the linear solvation energy relationships (LSER). ${ }^{7,8}$ Specifically characteristic interaction constants measuring the cavity formation/dispersive interaction strength, dipolarity/polarizability, hydrogen bond donating acidity and hydrogen bond accepting basicity for RPLC stationary phases were estimated by using a LSER model that is similar to Eq. (3) given below. While these interaction strength constants are not as general as the widely used Rohrschneider/McReynolds constants for GLC phases', it has been shown that they are useful in choosing the best column for a given separation among a number of nominally equivalent columns and columns with different bonded functionality. This model has also been used to characterize retention properties of carbon fiber in $\mathrm{GC}^{10}$, silica and 
alumina as the stationary phase in normal phase LC ${ }^{11}$, octadecylsiloxane-bonded silica and alumina ${ }^{12}$ and some cyanopropyl-bonded silica ${ }^{13}$ in RPLC.

Silica-based RP sorbents for SPE possess similar bonded functionalities as the RPLC stationary phases but the nature of base silica particles such as the specific surface area and pore size and volume is quite different from that of the silica particles used for RPLC stationary phases. It is thought that the characteristic interaction properties for a variety of RP sorbents will be useful for selection of the best sorbent for a given sample preparation. In the present work we use the multiple regression analysis based on the LSER methodology to estimate the characteristic interaction constants for various silica-based RP sorbents.

According to the LSER formalism of Abraham ${ }^{14}$, logarithmic capacity factors $\left(k^{\prime}\right)$ of the solutes for gas chromatographic retention can be correlated via the use of the following equation:

$$
\log k^{\prime}=c+l \log L^{16}+r R_{2}+s \pi_{2}^{*}+b \Sigma \beta_{2}+a \Sigma \alpha_{2}
$$

$c$ denotes the value of SP when all the other terms in the equation are zero. The solute descriptors are $R_{2}$, an excess molar refraction, $\pi_{2}{ }^{*}$, the solute dipolarity/ polarizability, $\Sigma \alpha_{2}$, the effective hydrogen bond donor acidity, $\Sigma \beta_{2}$, the effective $H B$ acceptor basicity, and $\log L^{16}$, the gas-hexadecane partition coefficient at $298 \mathrm{~K} .{ }^{15}$ The coefficients or constants, $c, l, r, s, b$ and $a$ in Eq. (1) are found by the method of multiple linear regression analysis. These constants can be identified with interaction characteristics of the particular system under study, more specifically the differences in interaction properties between the mobile and stationary phase. In the case of gas chromatography, the interactions in the mobile phase are zero and hence the coefficients in Eq. (1) can be regarded as characteristics of the particular stationary phase under study. The $a$ and $b$ coefficients, being complementary to the solute hydrogen bond acidity and basicity, represents the stationary phase hydrogen bond basicity and acidity, respectively. The $s$ coefficient is related to the stationary phase dipolarity/polarizability. The $l$ coefficient is related to a combination of dispersive interactions and the cavity effect and essentially measures the ability of the phase to distinguish between or to separate homologs. The $r$ coefficient refers to the ability of the phase to interact with the solute $\pi$ - and n-electron pairs and provides an indication of polarizability.

Recently Poole and coworkers ${ }^{16,17}$ determined retention characteristics of an octadecylsiloxane- and a cyanopropylsiloxane-bonded silica based sorbent by applying an equation similar to Eq. (1) to the high pressure liquid chromatographic capacity factors or to breakthrough volumes on the extraction cartridge. The sorption properties so determined are properties of the combination of the sorbent and sorbed mobile phase components since the sorbents are always modified by the sorbed mobile phase components in the sample or the mobile phase. Since the sorbent is usually used with solvents adsorbed on it, these sorption properties are useful to characterize sorption behavior of the sorbent in actual extractions of samples.

In the present work we measured capacity factors $\left(k^{\prime}\right)$ of a set of test solutes on GC columns packed with alkylsiloxane-, phenylsiloxane- and cyanopropylbonded reversed-phase sorbents for solid phase extraction and estimated the characteristic interaction constants of these sorbents by applying the LSER, Eq. (1), to $\log k^{\prime}$ data of the solutes. The interaction constants determined in this work using the GC retention data are intrinsic properties of the sorbents, not modified by sorbed sample solvents. Eq. (1) and these native interaction constants for sorbents can be utilized in designing new sorbents with different sorption properties.

\section{Experimental}

Reversed-phase sorbents investigated were either from Waters (Milford, USA) or Supple (Bellefonte, USA) and were packed in High Efficiency Grade stainless-steel type 316 tubing $\left(1 / 8^{\prime \prime}\right.$ i.d. $\left.\times 1 \mathrm{~m}\right)$ (Alltech Associates Inc., Deerfield, USA). Physical characteristics of the sorbents are listed in Table 1.

GC was performed with a DS 6200 gas chromatograph (Donam Systems Inc., Seoul, Korea) with a flame

Table 1 Characteristics of the sorbents as supplied by the manufacturers

\begin{tabular}{|c|c|c|c|c|c|c|c|c|}
\hline No. & Sorbent & Bonded ligand & $\begin{array}{c}\text { Carbon } \\
\text { loading, } \\
\%\end{array}$ & $\begin{array}{c}\text { Surface } \\
\text { area/ } \\
\mathrm{m}^{2} \mathrm{~g}^{-1}\end{array}$ & $\begin{array}{l}\text { Bonding } \\
\text { density }^{\mathrm{a}} / \\
\mu \mathrm{mol} \mathrm{m} \mathrm{m}^{-2}\end{array}$ & $\begin{array}{c}\text { Pore } \\
\text { size } / \\
\AA\end{array}$ & $\begin{array}{c}\text { Particle } \\
\text { size } / \\
\mu \mathrm{m}\end{array}$ & $\begin{array}{l}\text { End- } \\
\text { capped }\end{array}$ \\
\hline I & Sep-Pak C18 & monofunctional $\mathrm{C}_{18}$ & $(0.037)^{b}$ & 320 & 1.85 & 125 & $55-105$ & yes \\
\hline II & Sep-Pak t18 & trifunctional $\mathrm{C}_{18}$ & $(0.053)$ & 320 & 3.15 & 125 & $37-55$ & yes \\
\hline III & Sep-Pak C8 & monofunctional $\mathrm{C}_{8}$ & $9(0.028)$ & 320 & 2.67 & 125 & $37-55$ & yes \\
\hline IV & Sep-Pak CN & difunctional cyanopropyl & $6.5(0.020)$ & 320 & 3.80 & 125 & $55-105$ & yes \\
\hline V & Supelcoclean LC-Ph & phenyl & $5.3(0.011)$ & 500 & 1.59 & 60 & 40 & no \\
\hline
\end{tabular}

a. Bonding densities were calculated using an equation by Berendsen and de Galan. ${ }^{23}$ b. In parentheses are values of $\%$ carbon $\mathrm{m}^{-2}$. 
Table 2 Properties of the test solutes

\begin{tabular}{lcccccllllll}
\hline \multicolumn{1}{c}{ Solute } & $\log L^{16}$ & $R_{2}$ & $\pi_{2}{ }^{*}$ & $\Sigma \beta_{2}$ & $\Sigma \alpha_{2}$ & \multicolumn{1}{c}{ Solute } & $\log L^{16}$ & $R_{2}$ & $\pi_{2}{ }^{*}$ & $\Sigma \beta_{2}$ & $\Sigma \alpha_{2}$ \\
\hline Pentane & 2.162 & 0.000 & 0.00 & 0.00 & 0.00 & Propiophenone & 5.081 & 0.804 & 0.95 & 0.51 & 0.00 \\
Hexane & 2.668 & 0.000 & 0.00 & 0.00 & 0.00 & 2-Phenylethanol & 4.628 & 0.811 & 0.91 & 0.64 & 0.30 \\
Heptane & 3.173 & 0.000 & 0.00 & 0.00 & 0.00 & Benzyl alcohol & 4.221 & 0.803 & 0.87 & 0.56 & 0.33 \\
Nonane & 4.182 & 0.000 & 0.00 & 0.00 & 0.00 & Phenol & 3.766 & 0.805 & 0.89 & 0.30 & 0.60 \\
Methanol & 0.970 & 0.278 & 0.44 & 0.47 & 0.43 & Chlorobenzene & 3.657 & 0.718 & 0.65 & 0.07 & 0.00 \\
Ethanol & 1.485 & 0.246 & 0.42 & 0.48 & 0.37 & Nitrobenzene & 4.557 & 0.871 & 1.11 & 0.28 & 0.00 \\
1-Propanol & 2.031 & 0.236 & 0.42 & 0.48 & 0.37 & Benzene & 2.786 & 0.610 & 0.52 & 0.14 & 0.00 \\
1-Butanol & 2.601 & 0.224 & 0.42 & 0.48 & 0.37 & Toluene & 3.325 & 0.601 & 0.52 & 0.14 & 0.00 \\
Diethyl ether & 2.015 & 0.041 & 0.25 & 0.45 & 0.00 & Bromobenzene & 4.041 & 0.882 & 0.73 & 0.09 & 0.00 \\
Dibutyl ether & 3.924 & 0.000 & 0.25 & 0.45 & 0.00 & Ethylbenzoate & 5.075 & 0.689 & 0.85 & 0.46 & 0.00 \\
Nitromethane & 1.892 & 0.313 & 0.95 & 0.25 & 0.06 & Aniline & 3.993 & 0.955 & 0.96 & 0.41 & 0.26 \\
Propanone & 1.696 & 0.179 & 0.70 & 0.49 & 0.04 & -Chlorophenol & 4.775 & 0.915 & 1.08 & 0.20 & 0.67 \\
Butanone & 2.287 & 0.166 & 0.70 & 0.51 & 0.00 & $p$-Toluidine & 4.452 & 0.923 & 0.95 & 0.45 & 0.23 \\
Methyl acetate & 1.911 & 0.142 & 0.64 & 0.45 & 0.00 & Phenetole & 4.242 & 0.681 & 0.70 & 0.32 & 0.00 \\
Ethyl acetate & 2.314 & 0.106 & 0.62 & 0.45 & 0.00 & Benzaldehyde & 4.008 & 0.820 & 1.00 & 0.39 & 0.00 \\
Acetophenone & 4.501 & 0.818 & 1.01 & 0.48 & 0.00 & & & & & & \\
\hline
\end{tabular}

Table 3 Regression coefficients in Eq. (1) for the sorbents

\begin{tabular}{ccccccccc}
\hline Sorbent & $c$ & $l$ & $r$ & $s$ & $b$ & $a$ & $\rho^{\mathrm{a}}$ & SD $^{\mathrm{b}}$ \\
\hline I & $-0.55(0.02)$ & $0.46(0.01)$ & $0.08(0.05)$ & $0.10(0.04)$ & $0.38(0.04)$ & $0.11(0.04)$ & 0.999 & 0.03 \\
II & $-0.58(0.03)$ & $0.43(0.09)$ & NS & $0.12(0.03)$ & $0.10(0.05)$ & NS & 0.996 & 0.04 \\
III & $-0.45(0.02)$ & $0.37(0.01)$ & NS & $0.10(0.02)$ & $0.25(0.04)$ & $0.08(0.03)$ & 0.997 & 0.03 \\
IV & $-0.96(0.06)$ & $0.43(0.02)$ & $-0.42(0.11)$ & $0.74(0.11)$ & $0.25(0.11)$ & $0.71(0.09)$ & 0.991 & 0.08 \\
V & $0.05(0.07)$ & $0.29(0.02)$ & NS & $0.21(0.08)$ & $1.60(0.13)$ & $0.75(0.11)$ & 0.982 & 0.11 \\
\hline
\end{tabular}

a. Correlation coefficient. b. Regression standard deviation. c. Not significant.

ionization detector, maintaining the thermostat temperature within $\pm 0.1^{\circ} \mathrm{C}$ and carrier gas flow rate within $\pm 0.02 \mathrm{ml} \mathrm{min}^{-1}$. Analyte retention times were measured with an HP 3396 integrating recorder. The column dead time was determined using three successive $n$-alkanes according to the method described in the literature. ${ }^{18}$ The column temperature was maintained at $150^{\circ} \mathrm{C}$ and the carrier gas (helium) flow rate was varied from 7 to $14 \mathrm{ml} \mathrm{min} \mathrm{m}^{-1}$. The analyte was injected either as solution in pentane $(\mathrm{ca} .0 .1 \% \mathrm{v} / \mathrm{v})$ or as headspace vapor. Injection volumes were $1 \mu \mathrm{l}$ for solution and $100 \mu \mathrm{l}$ for vapor. At least duplicate measurements of retention times were performed for all analytes.

All the analytes were reagent grade from Aldrich (Milwaukee, USA) and were used without further purification.

\section{Results and Discussion}

The parameters values of 31 solutes of widely varying physicochemical properties are given in Table 2 . Results of application of Eq. (1) to measured logarithmic capacity factor values of the test solutes on the five RP sorbents are summarized in Table 3, where we give the characteristic constants in Eq. (1), together with the overall standard deviation in $\log k^{\prime}$ (SD) and the overall correlation coefficient $(\rho)$. As judged by the values of $\mathrm{SD}$ and $\rho$, the regression equations for the five sorbents are of excellent quality.

Inspection of Table 3 yields information about interaction behavior of the sorbents. The signs of all the constants are positive (except $r$ for Sep-Pak $\mathrm{CN}$ ), indicating that all types of interactions of the sorbent with solute lead to retention in the sorbent. For nonpolar sorbents such Sep-Pak C18, tC18 and C8, the $l$ constant is the largest, indicating that the cavity/ dispersive interactions term is the dominant term favoring retention on the sorbent. Other interaction terms play lesser roles in retention with relative contribution in the order: interactions with type A HB interactions (the $b$ constant)>type B HB interactions (the $a$ constant) $\approx$ dipolar interactions (the $s$ constant) $>$ lone pair interactions (the $r$ constant). Quite large HB acidity (the $b$ constant) and minor but still significant dipolarity (the $s$ constant) and HB basicity (the $a$ constant) of these nonpolar sorbents are believed to be due to the residual surface silanol groups on the silica support. It is noted that Sep-Pak tC18, which has the greatest surface coverage, show the smallest values of these coefficients.

For the polar sorbent Sep-Pak $\mathrm{CN}$, the $s$ and $a$ 
Table 4 Individual solute-sorbent interaction terms contributing to $\log k^{\prime}$ in Eq. (1)

\begin{tabular}{lcccccccc}
\hline Solute & Sorbent & $l \log L^{16}$ & $r R_{2}$ & $s \pi_{2}{ }^{*}$ & $b \Sigma \beta_{2}$ & $a \Sigma \alpha_{2}$ & $c$ & $\log k^{\prime}$ \\
\hline Toluene & I & 1.53 & 0.05 & 0.05 & 0.05 & 0 & -0.55 & 1.13 \\
& II & 1.43 & 0 & 0.06 & 0.01 & 0 & -0.58 & 0.93 \\
& III & 1.23 & 0 & 0.05 & 0.04 & 0 & -0.45 & 0.87 \\
1-Butanol & IV & 1.43 & -0.25 & 0.38 & 0.04 & 0 & -0.96 & 0.64 \\
& V & 0.96 & 0 & 0.11 & 0.22 & 0 & 0.05 & 1.35 \\
& I & 1.20 & 0.02 & 0.04 & 0.18 & 0.04 & -0.55 & 0.93 \\
Butanone & II & 1.12 & 0 & 0.05 & 0.05 & 0 & -0.58 & 0.64 \\
& III & 0.96 & 0 & 0.04 & 0.12 & 0.03 & -0.45 & 0.70 \\
& IV & 1.12 & -0.09 & 0.31 & 0.12 & 0.26 & -0.96 & 0.76 \\
& V & 0.75 & 0 & 0.09 & 0.77 & 0.28 & 0.05 & 1.94 \\
& I & 1.05 & 0.01 & 0.07 & 0.19 & 0 & -0.55 & 0.78 \\
Nitrobenzene & II & 0.98 & 0 & 0.08 & 0.05 & 0 & -0.58 & 0.54 \\
& III & 0.85 & 0 & 0.07 & 0.13 & 0 & -0.45 & 0.59 \\
& IV & 0.98 & -0.07 & 0.52 & 0.13 & 0 & -0.96 & 0.60 \\
& V & 0.66 & 0 & 0.15 & 0.82 & 0 & 0.05 & 1.68 \\
& I & 2.10 & 0.07 & 0.11 & 0.11 & 0 & -0.55 & 1.83 \\
& II & 1.96 & 0 & 0.13 & 0.03 & 0 & -0.58 & 1.54 \\
& III & 1.69 & 0 & 0.11 & 0.07 & 0 & -0.45 & 0.91 \\
& IV & 1.96 & -0.37 & 0.82 & 0.07 & 0 & -0.96 & 1.52 \\
& V & 1.32 & 0 & 0.23 & 0.45 & 0 & 0.05 & 2.05 \\
\hline
\end{tabular}

constants are the largest, followed by the essentially equal $l$ and $r$ constants. The $b$ constant is the smallest but still significant. On this polar sorbent dipolar interactions and type $\mathrm{B}$ HB interactions are major contributors to the solute retention on the sorbent. Large $s$ and $a$ constants are due to the large dipolarity and $\mathrm{HB}$ basicity of the cyanopropyl group (the $\pi^{*}$ and $\beta$ values of unbound analog, butyronitrile, are 0.71 and 0.40 , respectively ${ }^{19}$ ). The sign of the $r$ constant is unexpectedly negative, indicating that the sorbent interactions with the solute $\mathrm{n}$ - and $\pi$-electrons act to decrease sorption. We do not have an explanation for the negative $r$ constant, but negative $r$ constants have been observed for some sorbent coatings on chemical microsensors for vapor sorption. ${ }^{20}$

Peculiar interaction behavior represented by a very large $b$ and $a$ constants for phenyl-bonded sorbent (LC$\mathrm{Ph}$ ) is surprising in view of the nompolar nature of the bonded phenyl group. The insignificant $r$ constant is also unexpected in view of the fact that a sorbent with a substantial proportion of phenyl groups should have a greater $r$ constant than alkyl and cyano-bonded sorbents. Unusually high HB acidity and HB basicity of the phenylbonded sorbent is believed to be due to a greater number of residual surface silanol groups left on the silica support due to low bonding density and/or to $\mathrm{HB}$ active impurities in the bare silica. It is noted that this sorbent is not end-capped and the bonding density is very low. It is likely that strong orientational HB interactions between residual silanol groups on the sorbent and the solute may dampen the potential for weak interactions with $\mathrm{n}$ - and $\pi$-electrons on the solute and the sorbent, leading to the insignificant $r$ value. This speculation needs to be substantiated by measuring $k^{\prime}$ on a column packed with a different lot of the LC-Ph sorbent.
Given the constants of the sorbents and the solute parameters, the individual terms in Eq. (1) can be calculated to estimate the individual interactions contributing the sorption process (Table 4). The sorbent Sep-Pak CN is selective for both dipolar and $\mathrm{HB}$ acidic solutes. The sorbent $\mathrm{LC}-\mathrm{Ph}$ is the most selective for $\mathrm{HB}$ basic solutes. Nitrobenzene is the most polar solute with only moderate HB basicity, 1-butanol is both HB acidic and basic while butanone is a strongly $\mathrm{HB}$ basic solute. The sorbent Sep-Pak CN sorbs nitrobenzene very strongly. This sorbent also sorbs the HB acid 1butanol quite selectively. It is noteworthy that the sorbent LC-Ph sorbs all types of solutes most strongly despite the fact that its bonding density is the lowest among the sorbents investigated. It is most likely that strong sorption of the solute on this sorbent is due to interactions of the solute with a large amount of the residual surface silanol groups on the silica support. The sorbent is not end-capped and has a very high specific surface area.

In all cases in Table 4, the $l \log L^{16}$ term contributes most significantly to the overall sorption but does not have a large effect on the selectivity among the sorbents. The $l \log L^{16}$ term is comprised of contributions from the cavity formation which disfavors sorption and dispersion interactions which favor sorption. It has, however, been shown ${ }^{21}$ that the main contribution to this term is solute-solvent dispersion and cavity contributions are not very different in nearly all liquid phases except water. Dispersion interactions always occur in the sorption of the solute and these are not particularly selective, with the exception of solutes having very different $\log L .^{16}$ It is noted that even though the bonding densities of the sorbent Sep-Pak tC18 are much greater than that of Sep- 
Pak C18 the magnitudes of the $l$ constants for $\mathrm{tC} 18$ and C18 are similar to each other. The magnitude of the $l$ constant for the sorbent Sep-Pak C8, whose bonding density is higher than that for the sorbent Sep-Pak C18, is smaller than that for the sorbent Sep-Pak C18. This is somewhat unexpected in view of the fact that the extent of dispersion interactions of the sorbent with the solute is expected to increase with increased density of the bonded ligand. Snyder and co-workers ${ }^{4,22}$ showed that retention in high performance RPLC increases with an increase in the amount (volume) and the surface area of the bonded phase. Stationary phase volume decrease in the order: $\mathrm{C} 18>\mathrm{C} 8$, but bonded-phase surface area (and bonding density) tends to increase from $\mathrm{C} 18$ to $\mathrm{C} 8$. It has been suggested ${ }^{6}$ that the $\% \mathrm{C} \mathrm{m}^{-2}$ of the bonded ligand, rather than bonding density in $\mu \mathrm{mol} \mathrm{m} \mathrm{m}^{-2}$, better represents the combination of volume and surface area of the bonded phase. The values of $\% \mathrm{C} \mathrm{m}^{-2}$ for the alkylbonded sorbents are decreasing in the order: tC18> $\mathrm{C} 18>\mathrm{C} 8$ (see Table 1), and generally so are the values of the $l$ constants.

In conclusion, interaction constants of a given sorbent provide specific information on the selectivity and general retention properties of the sorbent concerned. Information obtainable from LSER (Eq. (1)) can be useful for designing of new sorbents.

This work was supported by a grant (No. 951-0304-045-2) from the Korea Science and Engineering Foundation.

\section{References}

1. C. F. Poole and S. K. Poole, "Chromatography Today", Elsevier, Amsterdam, 1991.

2. M. F. Delaney, A. N. Papas and M. J. Walters, $J$. Chromatogr., 410, 31 (1987).
3. R. M. Smith and S. L. Miller, J. Chromatogr., 464, 297 (1989).

4. P. E. Antle, A. P. Goldberg and L. R. Snyder, $J$. Chromatogr., 321, 1 (1985).

5. H. Engelhardt, H. Low and W. Gotzinger, $J$. Chromatogr., 544, 371 (1991).

6. J. H. Park, J. J. Chae, T. H. Nah and M. D. Jang, J. Chromatogr. A, 664, 149 (1994).

7. M. J. Kamlet, J. L. M. Abboud and R. W. Taft, Prog. Phys. Org. Chem., 13, 591 (1981).

8. M. J. Kamlet and R. W. Taft, Acta Chem. Scand., B39, 611 (1985).

9. W. O. McReynolds, J. Chromatogr. Sci., 8, 685 (1970).

10. J. H. Park, Y. K. Lee and J. B. Donnet, Chromatographia 33, 154 (1992).

11. J. H. Park and P. W. Carr, J. Chromatogr., 465, 123 (1989).

12. J. H. Park, Bull. Korean Chem. Soc., 11, 568 (1990).

13. J. H. Park, M. D. Jang and S. T. Kim, Bull. Korean Chem. Soc., 11, 297 (1990).

14. M. H. Abraham, Chem. Soc. Rev., 22, 73 (1993).

15. M. H. Abraham, J. Phys. Org. Chem., 6, 660 (1993).

16. K. G. Miller and C. F. Poole, J. High Resolut. Chromatogr., 17, 125 (1994).

17. D. S. Seibert and C. F. Poole, J. High Resolut. Chromatogr., 18, 226 (1995).

18. X. Guardino, J. Albaiges, G. Firpo, R. V. Rodriguez and M. Gassiot, J. Chromatogr., 131, 41 (1977).

19. Y. Marcus, Chem. Soc. Rev., 93, 409 (1993).

20. M. H. Abraham, J. Andonian-Haftvan, G. S. Whiting, A. Leo and R. S. Taft, J. Chem. Soc., Perkin Trans 2, 1994, 177.

21. M. H. Abraham, G. S. Whiting, R. M. Doherty and W. J. Shuley, J. Chem. Soc., Perkin Trans 2, 1990, 1851.

22. P. E. Antle and L. R. Snyder, $L C, 2,840$ (1984).

23. G. E. Berendsen and L. de Galan, J. Chromatogr., 1, 561 (1978).

(Received June 24, 1996)

(Accepted July 24, 1996) 